W. 1. Prokiny (fid.), Classical comditioning. vels York: Appletoncentury-frofts, 196.5. Pp. 118-147.

LAI I VIR. H. Conditioned suppression in rats and the effect of phamacological agents thcrcon. Psychopharmacologia, 1963, 4. 311.325 .

1.1 Al: R. C. Motivational propertics of the $C S$ in conditioned suppression. Psychonomic Science, $1966.5,43$.

LYON. D. O. Conditioned suppression: Operant variables and aversive control. Psychological Record, 1968, 18, 317.338.

MAILR, S.. SILLIGMAN, M., \& SOLOMON, R. Pavlovian fear conditioning and learned helplessness: liffects on escape and avoidance bchavior of (a) the (S-L:S contingency and (b) the independence of the $\mathrm{L} S \mathrm{~S}$ and voluntary

\title{
Effects of social isolation until adulthood on maternal behavior in guinea pigs*
}

\author{
JEFFREY J. STERN and BONITA M. HOFFMAN \\ University of Michigan, Dearborn, Mich. 48128
}

Five female guinea pigs were separated from their mothers at birth and reared in social isolation until adulthood. Following impregnation and parturition the maternal behavior of these females was compared to that of a mother-reared group. No differences were found on nursing time, nursing posture, pup-oriented responses, or on weight gain of offspring.

Thoman \& Arnold (1968) developed a method for maintaining newborn rats in an incubator. Using this technique, 17 Holtzman albino females were reared in isolation for Days 1-25. For Days 26-84 the females were housed individually. On Day 85 males were placed with the isolates and left until the females were found to be pregnant. Following parturition the maternal behavior of these females was found to differ little from that of females reared in groups.

Arling \& Harlow (1967) separated female rhesus monkeys from their mothers at birth and reared them in social isolation for 10-24 months. These females, in contrast to the rat isolates, were found to be deficient in the basic patterns of maternal behavior. In general, the rhesus monkey isolates were indifferent or abusive to their offspring.

The purpose of the present study was to examine the effects of social isolation on the maternal behavior of a third mammal, the guinea pig.

* This research was funded by a University of Michigan Office of Research Administration grant to J.J.S. The authors wish to thank Michael Murphy for his valuable assistance during the course of the experiment. responding. In B. Campbell and R. Church (lids.), Punishment and alersive behavior. New York: Appleton-Century-Crofts, 1969. Pp. 299-342.

MILI.NSON, J. R., \& HENDRY, D. $P$. Quantification of response suppression in conditioned anxicty training. Canadian Joumal of P'sychology, 1967, 21, 242-252.

STFIN, L., SIDMAN, M., \& BRADY, J. Some effects of two temporal variables on conditioned suppression. Journal of the Experimental Analysis of Behavior, 1958, 1, 153-162.

ZILL, N. The effects of temporal uncertainty on conditioned suppression and preference for warned shock in rats. Unpublished doctoral dissertation, The Johns Hopkins University, 1967. testing taking place from October 5, 1969 to January 11, 1970. The laboratory's overhead lights were turned on during most of the daylight hours and during night-time testing.

\section{SUBJECTS \\ Isolates}

Nine of 16 females survived being separated from their mothers at birth and reared in social isolation for 3 months. The female neonates were released from the foetal membranes, dried by the $E$, and placed in individual plastic boxes (12 $12 \times 12$ in.). Each box had a heating pad on the floor. Flood lamps (150-W) were placed $3 \mathrm{ft}$ above the boxes and were constantly turned on during the pup's first week of life. During the first 2 weeks, the pups were periodically hand-fed Mead Johnson Enfamil. In addition, a dish of Enfamil was present in each box at all times. Between Days 21-25 the females were placed in rabbit cages that were lined with aluminum foil. Starting at 95-111 days, the females were manually examined each night for lordosis. Following the first display of the lordotic response, the female was given a sexually potent male for the night. Until adulthood, therefore, these females had neither seen nor had physical contact with a congener. Seven of the nine females became pregnant. Two of the seven died during the experiment, leaving an experimental group of five.

\section{Mother Reared}

Ten pregnant guinea pigs were purchased from a commercial breeder. Eight of these females survived pregnancy and parturition and constituted the mother-reared group. PROCEDURE

The Ss and their offspring were observed animals were on a natural light cycle with

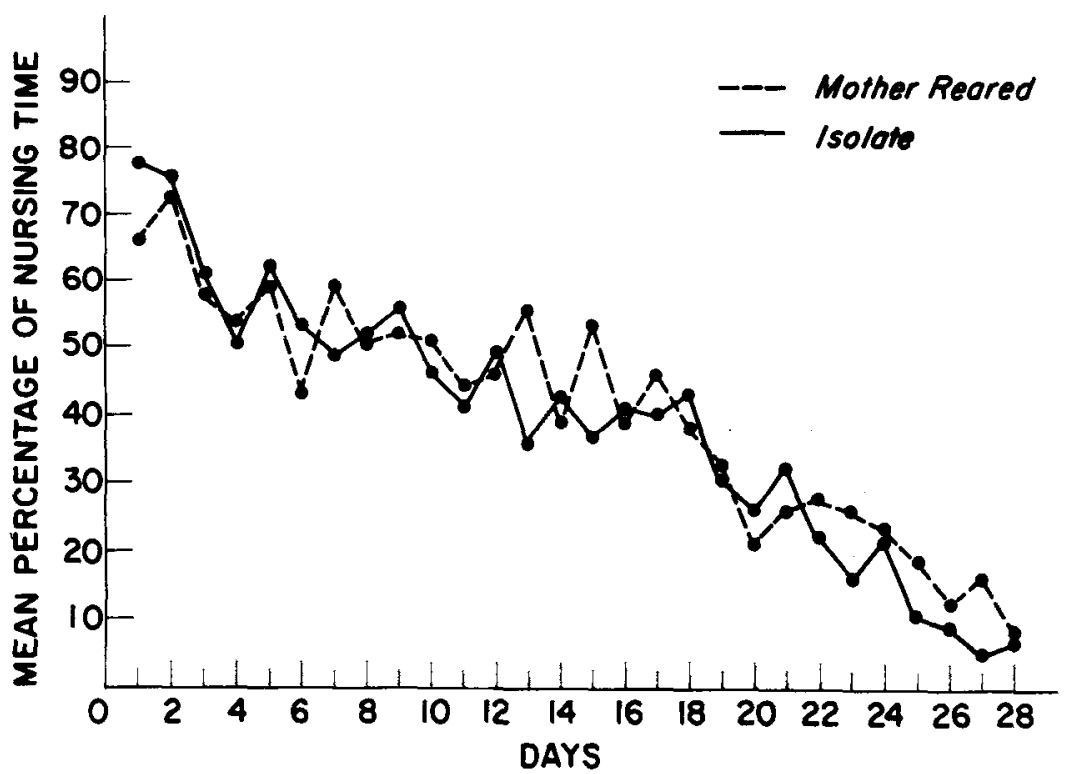

Fig. 1. Mean percentage of nursing time over the 4 weeks of observation. 


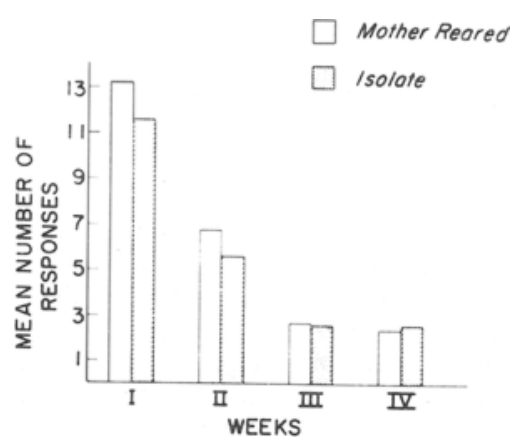

Fig. 2. Mean number of anogenital responses during each of the 4 weeks of observation.

in their home cages $30 \mathrm{~min}$ per day for Days 1-28. The $30 \mathrm{~min}$ of observation consisted of three 10-min observation periods separated by intervals of 4 to $13 \mathrm{~h}$. During each period, E recorded the time the female was in the nursing posture and the number of times the female licked the pups' anogenital regions. During the first days of life, the guinea pig is unable to urinate or defecate, and it is believed that the stimulation provided by the mother's perianal licking induces the pup to void (Beach, 1966). In addition to these daily observations, the pups were weighed on the day of birth and every 7 days thereafter for the next 4 weeks.

\section{RESULTS}

Qualitative Observations

It was not possible to differentiate the mother-reared from the isolate group on the basis of maternal behavior. Neither nursing posture nor mother-pup interactions were distińguishable.

Quantitative Observations

The five isolate females had 14 pups
$(M=2.8)$, while the eight mother-reared females had $21(\mathrm{M}=2.6)$. One of the mother-reared pups was stiliborn and one died during the experiment, leaving 19 pups at Day 29.

Figure 1 shows the mean percentage of nursing time for Days 1-28. Immediately following parturition, both groups were characterized by high percentages of nursing time (70\%). By Day 14 this percentage was reduced to $50 \%$, and by Day 28 the females were spending only $10 \%$ of the observed time nursing. Mann-Whitney U tests over each of the 4 weeks reveal no significant differences.

Figure 2 depicts the mean number of anogenital responses by the females. During Week 1 both groups had approximately 12 responses. The number of responses was reduced to six during Week 2 and to three during Weeks 3 and 4. Mann-Whitney $U$ tests reveal no differences for the 4 weeks.

The mean weights of the pups are shown in Fig. 3. The pups of the isolate females weighed more on Day $1(6 \mathrm{~g})$. The difference was maintained over the 4 weeks reaching $15 \mathrm{~g}$ on Day 29. For Days 1,8 , 15,22 , and 29 , t tests reveal a difference only for Day $29(\mathrm{p}<.05)$.

\section{DISCUSSION}

Social isolation until adulthood has no effect on the maternal behavior of the guinea pig. There were no differences between the isolate and mother-reared females on nursing time (Fig. 1), nursing posture, pup-oriented responses (Fig. 2), or on the weight gain of the offspring (Fig. 3).

Following early social isolation the guinea pig and the rat display typical maternal behavior, while the rhesus monkey exhibits an altered pattern. The increased role of early social experience for

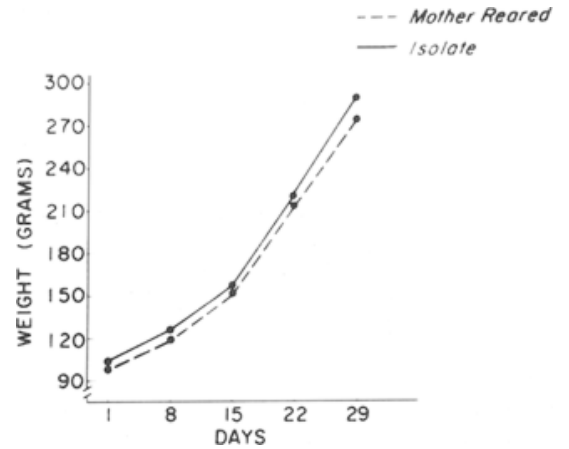

Fig. 3. Mean weight of the pups for Days 1-29.

the rhesus monkey parallels the findings for sexual behavior. Following early social isolation, female guinea pigs display typical mating behavior (Harper, 1968), while rhesus monkeys do not (Arling \& Harlow, 1967). To date, no physiological explanation has been put forward to account for these species differences.

\section{REFERENCES}

ARLING, G. L., \& HARLOW, H. F. Effects of social deprivation on maternal behavion of rhesus monkeys. Journal of Comparative \& Physiological Psychology, 1967, 64, 371-377. BEACH, F. A. Ontogeny of "coitus-related" reflexes in the female guinea pig. Proceedings of the National Academy of Science, 1966, 56, 526-533.

HARPER, L. V. The effects of isolation from birth on the social behaviour of guinea pios in adulthood. Animal Behaviour, 1968, 16 , 58-64.

THOMAN, E. B., \& ARNOLD, W. J. Effects of incubator rearing with social deprivation on maternal behavior in rats. Journal of Comparative \& Physiological Psychology, $1968,65,441-446$. 